\title{
A DATA COLLECTION PROTOCOL WITH ENERGY EFFICIENCY AND DELAY AWARE IN WIRELESS SENSOR NETWORKS
}

\author{
Md Masood Huzaif ${ }^{1}$, Rajashree V Biradar ${ }^{2}$ \\ ${ }^{1}$ Computer Network and Engineering, Ballari Institute of Technology and Management, Bellary, India \\ ${ }^{2}$ Computer Network and Engineering, Ballari Institute of Technology and Management, Bellary, India
}

\begin{abstract}
The various researches on open vehicle routing (OVR) problems endeavour that it is based on same assumptions and constraints as that of sensor networks. Thus, it may be feasible to adapt these techniques so that they will provide valuable solutions to certain tricky problems in the wireless sensor network (WSN) domain. To demonstrate that this approach is feasible, one data collection protocol called EDAL, which stands for Energy-efficient Delay-aware Lifetime-balancing data collection, is defined. Therefore, both a centralized heuristic to reduce its computational overhead and a distributed heuristic to make the algorithm scalable for large-scale network operations is defined. The applications in WSN are evolving, so congestion control remains an open and, in several cases, a critical problem. Two general approaches seem to be the most prominent for its solution: traffic control and resource control. A dynamic scheme capable of bridging these two methods for congestion control and provide the best solution
\end{abstract}

Keywords: Data Collection, Energy-Efficiency, Routing Protocols, Wireless Sensor Networks.

\section{INTRODUCTION}

The Wireless Sensor Network (WSN) are defined to be a network of autonomous, battery-powered devices in which data is generated by one or more source nodes and routed to one or more sink nodes. It consists of nodes deployed in hostile or remote environments for a wide range of applications, such as environment monitoring, scientific observation, emergency detection, and many more. In these applications, prolonging the lifetime of WSN and guaranteeing packet delivery delays are critical for achieving acceptable quality of service.

In many sensing applications the source nodes deliver packets to sink nodes via multiple hops. This leads to the problem on how to find routes to the sink that enable all packets to be delivered in required time frames, while simultaneously taking into account factors such as energy efficiency and load balancing. Many previous research efforts have tried to achieve tradeoffs in terms of delay, energy cost, and load balancing for such data collection tasks. Our main motivation for this work is from the insight that various research efforts on open vehicle routing (OVR) problems are usually based on similar assumptions and constraints compared to sensor networks. Specifically, in OVR research on goods transportation, the objective is to spread the goods to customers in finite time with the minimal amount of transportation cost. If we treat packet delays as delivery time of goods, and energy cost as delivery cost of goods, it may be possible to exploit research results in one domain to stimulate the other.

Motivated by this observation, an Energy-efficient DelayAware Lifetime-balancing data collection protocol called EDAL is defined. Specifically, EDAL is formulated by treating energy cost in transmitting packets in WSNs in a similar way as delivery cost of goods in OVR and by treating packet latencies similar to delivery deadlines. It proves that the problem addressed by EDAL is NP-hard. The algorithm designs also take into account load balancing of individual nodes to maximize the system lifetime. Finally, it integrates the algorithm with compressive sensing.

More specifically, our major contributions are as follows.

- To provide the data collection protocol called EDAL, which employs the techniques developed for OVR in operations research to find the minimum cost routes to deliver packets within the specified deadline.

- To improve the performance and scalability in the Wireless Sensor Network.

- To ensure that all packets reach the destination node under the constraint that no packet deadline is violated.

\section{LITERATURE SURVEY}

Dantzig and Ramser (1959) were the first to introduce the "Open Vehicle Routing Problem (OVRP)", modelling on how to find routes between a depot and customers with given demands so that the transportation cost is minimized with the involvement of the minimal number of vehicles, while satisfying capacity constraints. The current OVRP models, however, are immensely different from the one introduced by Dantzig and Ramser (1959), as they increasingly aim to incorporate real-life complexities, such as for instance time-dependent travel times, time windows for pickup and delivery, and input information that changes dynamically over time. These features bring along substantial complexity. 
The vehicle routing problem (VRP) is a well-known NPhard problem in operational research and thus exact algorithms are only efficient for small problem instances. Heuristics and metaheuristics are often more suitable for practical applications, because real-life problems are considerably larger in scale.

With additional constraints, VRP can be further extended to solve different problems, where one of the most important is the vehicle routing problem with time windows (VRPTW). This problem occurs frequently in the distribution of goods and services, where an unlimited number of identical vehicles with predefined capacity serve a set of customers with demands of different time intervals (time windows). This led to the mixed routing and scheduling problems and asks for algorithms that also take temporal aspects into account.

The VRPTW is defined as "A number of vehicles is located at a single depot and must serve a number of geographically dispersed customers. Each vehicle has given a capacity. Each customer has given a demand and must serve within a specified time window." The objective is to minimize the total cost of travel. VRPTW tries to minimize the total transportation cost through the minimum number of vehicles, without violating any timing constraints in delivering goods.

If vehicles are not required to return back to the depot, and if the time windows are replaced by deadlines, VRPTW can be further extended to the open vehicle routing problem with time deadlines (OVRP-TD).

As an NP-hard problem, OVRP-TD has inspired many heuristics. Ozyurt et al. proposed the nearest insertion method, where the farthest node is chosen first to be connected with a route. Then, repeatedly, each selected node chooses the nearest neighbour that has not been assigned a route so far and connects itself to this neighbour. This procedure repeats until all customers are connected by routes.

Solomon [5] developed the push forward insertion heuristic (PFIH), which repeatedly selects the customer with the lowest additional insertion cost as the next node, until all customers are connected. Once initial routes have been found, various algorithms are developed to generate nearoptimal solutions based on simulated annealing, tabu search, or genetic programming.

Xiang et al. proposed a new data aggregation technique derived from CS to minimize the total energy consumption through joint routing and compressed aggregation. Mehrjoo et al. employed compressive sensing and particle swarm optimization algorithms to build up data aggregation trees and decrease communication rate. These two methods are different from EDAL in that they require all nodes to contribute sensing data during the data collection phase. On the other hand, Xiang et al. proposed random routing methods based on different network topologies to collect data from a subset of nodes, which is a similar application scenario as EDAL.
However, EDAL achieves better energy efficiency because it optimizes the number of constructed routes such that the total number of packets is decreased. It further compare the performance of EDAL with that reported in in the evaluation section to show that a better gain in energy efficiency is achieved because it exploits the topological requirements of compressive sensing.

Optimal Node Selection for Target Localization in Wireless Camera Sensor Networks. The goal of node selection is to optimize the tradeoff between the energy consumption of wireless camera sensor networks and the quality of target localization. Here it proposes a cooperative target localization algorithm, which is implemented by two phases: 1) target detecting phase and 2) target locating phase. For the target detecting phase, it develops a probing environment and adaptive sleeping (PEAS)-based density control algorithm to select the proper subset of deployed camera sensors for maintaining the desired density of nodes in the detecting mode. For the locating phase, it maps the node-selection problem into an optimization problem and then proposes an optimal node-selection algorithm to select a subset of camera sensors for estimating the location of a target while minimizing the energy cost.

Distributed Compressive Sampling for Lifetime Optimization in Dense Wireless Sensor Networks. Increasing network size poses significant data collection challenges, for what concerns sampling and transmission coordination as well as network lifetime. To tackle these problems in-network compression techniques without centralized coordination are becoming important solutions to extend lifetime. In this paper it considers a scenario in which a large WSN, based on ZigBee protocol, is used for monitoring .It proposes a new algorithm for in-network compression aiming at longer network lifetime. Our approach is fully distributed: each node autonomously takes a decision about the compression and forwarding scheme to minimizing the number of packets to transmit. Performance is investigated with respect to network size using data sets gathered by a real life deployment.

\section{SYSTEM ARCHITECTURE}

The system design contains 3 main parts, i.e., Configuration Manager Unit, Distributed Heuristic Unit, and Ant Colony Optimization Unit.

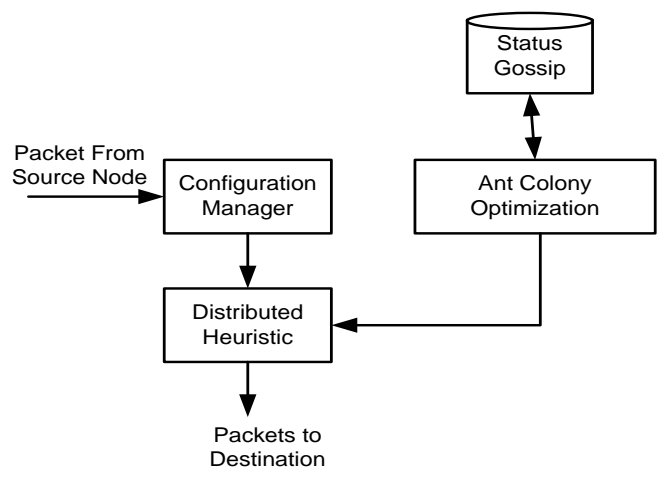

Fig 1: Architecture 
- Configuration Manger Unit: The configuration manager performs the process of monitoring and controlling the network devices. This component collects the packets from the networks, which are in-turn specified by the user.

- Distributed Heuristic Unit: The collected information then performs Distributed Routing using the Ant Colony Optimization algorithm. In Distributed routing, each node independently chooses the most energy efficient route to forward packets.

- Ant Colony Optimization Unit: In Ant Colony Optimization, each node sends forward ants spreading its current status, including its remaining energy level, toward its neighbour nodes within $\mathrm{H}$ hops. Meanwhile, the status data of nearby nodes is collected by each source node with the received backward ants. The Status Gossip is used to store the status information of various nodes.

\section{EXISTING SYSTEM}

The existing technique is known to be Energy Balanced Routing Protocol (EBRP).

The efficient routing protocols in Wireless Sensor Networks, is usually focused on two major parameters namely energy balance and energy efficiency. These two parameters are different attributes of routing techniques design goal. The energy-efficient routing protocol is trying to extend the network lifetime in terms of energy consumption, whereas the energy-balanced routing protocol aims to maximize the network lifetime through even and uniform energy consumption. Thus the EBRP fails to achieve Throughput, End-to-End Delay, in order to improve the Network Performance.

\subsection{Proposed Methodology}

It proposes both a centralized heuristic to reduce its computational overhead and a distributed heuristic to make the algorithm scalable for large-scale network operations. It also develop EDAL to be closely integrated with compressive sensing, an emerging technique that promises considerable reduction in total traffic cost for collecting sensor readings under loose delay bounds. Finally, it systematically evaluates EDAL to compare its performance to related protocols in both simulations and a hardware testbed.

And a dynamic scheme capable of bridging these two methods for congestion control and provide the best solution is also defined.

\section{CONCLUSION}

In here, defining EDAL, an Energy-efficient Delay Aware Lifetime-balancing protocol for data collection in wireless sensor networks, which is inspired by recent techniques developed for open vehicle routing problems with time deadlines (OVRP-TD) in operational research. The goal of
EDAL is to generate routes that connect all source nodes with minimal total path cost, under the constraints of packet delay requirements and load balancing needs. The lifetime of the deployed sensor network is also balanced by assigning weights to links based on the remaining power level of individual nodes. It proves that the problem formulated by EDAL is NP-hard. Therefore, a centralized heuristic to reduce its computational complexity is defined. Furthermore, we proposed, a hybrid scheme for congestion control in WSNs. This scheme attempts to complement the resource control method with traffic control. In particular, when resource controls in unable to be effectively applied in a specific network instance, the algorithm employs the traffic control method. The advantage of this hybrid solution lies on the fact that due to the frequent variations that take place in WSNs topologies and node placements, each node is able to figure out which congestion control method is the most appropriate to apply at any moment, giving priority to resource control that extends network lifetime as well as throughput.

\section{REFERENCES}

[1] B. Eksioglu, A. V. Vural, and A. Reisman, "The vehicle routing problem: A taxonomic review,"Comput. Ind. Eng., vol. 57, no. 4, pp. $1472-$ 1483, 2009.

[2] O. Bräysy and M. Gendreau, "Vehicle routing problem with time windows, Part I: Route construction and local search algorithms,"Transport. Sci., vol. 39, no. 1, pp. 104-118, Feb. 2005.

[3] Z. Ozyurt, D. Aksen, and N. Aras, "Open vehicle routing problem with time deadlines: Solution methods and an application," in Operations Research Proceedings 2005, ser. Operations Research Proceedings, H.-D.Haasis, H.Kopfer, and J.Schnberger, Eds. Berlin,Germany: Springer, 2006, vol. 2005, pp. 73-78.

[4] M. M. Solomon, "Algorithms for the vehicle routing and scheduling problems with time window constraints,'Oper. Res.,vol.35,no.2, pp. 254-265, 1987.

[5] L. Xiang, J. Luo, and A. Vasilakos, "Compressed data aggregation for energy efficient wireless sensor networks," inProc. 8th Annu. IEEE SECON, Jun. 2011, pp. 46-54.

[6] Yanjun Yao, Qing Cao and Athanasios V. Vasilakos, "EDAL: An Energy-Efficient, DelayAware, and Lifetime-Balancing Data Collection Protocol for Heterogeneous Wireless Sensor Networks"IEEE / ACM transaction on networking, no. 1, pp. 1-14, 2014

[7] L. Liu, X. Zhang, and H. Ma, "Optimal node selection for target localization in wireless camera sensor networks," IEEE Trans. Veh. Technol., vol. 59, no. 7, pp. 3562-3576, Sep. 2010.

[8] C. Caione, D. Brunelli, and L. Benini, "Distributed compressive sampling for lifetime optimization in dense wireless sensor networks," IEEE Trans. Ind. Inf., vol. 8, no. 1, pp. 30-40, Feb. 2012. 